\title{
Rapid Organoid Reconstitution by Chemical Micromolding
}

${ }^{*}$ Robert J Weber ${ }^{1,2,3}$, *Alec E Cerchiari ${ }^{1,4,5}$, Lucas S Delannoy ${ }^{5}$, James C Garbe ${ }^{1,6}$, Mark A LaBarge ${ }^{6}$, Tejal A Desai ${ }^{4,7}$, Zev J Gartner ${ }^{1,2,4}$

${ }^{*}$ Equal Contributions

${ }^{1}$ Department of Pharmaceutical Chemistry, University of California San Francisco, $60016^{\text {th }}$ St. Box 2280, San Francisco, CA $94158,{ }^{2}$ Chemistry and Chemical Biology Graduate Program, University of California, San Francisco, $60016^{\text {th }}$ St. Room 522, San Francisco, CA 94158, ${ }^{3}$ Medical Scientist Training Program, University of California, San Francisco, 513 Parnassus Ave, San Francisco, CA $94143{ }^{4}$ UC Berkeley - UCSF Group in Bioengineering, $17004^{\text {th }}$ St., Room 216, Box 0775, San Francisco, CA 94158, ${ }^{5}$ Laboratory of Stem Cell Bioengineering, Institute of Bioengineering, École Polytechnique Fédérale de Lausanne, Station 15, Bld. Al 1106, CH-1015 Lausanne ${ }^{6}$ Life Sciences Division, Lawrence Berkeley National Laboratory, 1 Cyclotron Road, Berkeley, CA $94720^{7}$ UCSF Bioengineering and Therapeutic Sciences, $17004^{\text {th }}$ St Room 216B Box 0775, San Francisco, CA 94158

\section{Contents}

Rapid Tissue Reconstitution via Chemical Micromolding

Contents

Materials and Methods

Oligonucleotide Sequences

MALDI Data

MALDI Traces

Figure S1 Tissue Coalescing in Absence of Capping Strand

Figure S2 Kinetics of Assembly

Figure S3 Scaled-up Chemical Micromolding

Figure S4 Double Labeling Cells

Table S1 Map of Capping Strand Adhesion and Sequences 


\section{Materials and Methods:}

\section{Synthesis of Fatty Acid-Modified Oligonucleotides and Labeling Cells with Single Stranded DNA}

The procedure used to synthesize fatty acid-modified oligonucleotides and label cells was previously described ${ }^{1}$. In brief, oligonucleotide strands were synthesized on an Expedite 8909 DNA synthesizer using reagents and protocols standard to the instrument. A terminal amine was incorporated either via modified phosphoramidite for the 5' amine (Glen Research 10-1906-90) or by utilizing a modified solid support for the 3 ' amine (Glen Research 20-2958). DNA was elaborated with the fatty acid membrane anchors lignoceric acid and palmitic acid for the adhesion and co-anchor strands respectively by condensation using carbodiimides. Fatty acid-modified strands of 20 bases for co-anchor and 100 bases for adhesion strands were purified from residual, unmodified oligonucleotides by reverse phase chromatography on an Agilent 1200 Series HPLC System using $100 \mathrm{mM}$ triethylamine acetate (TEAA, pH 7) $\mathrm{H}_{2} \mathrm{O} /$ acetonitrile as a mobile phase on a C8 column (Hypersil Gold, Thermo scientific) running a gradient between 8 and $95 \%$ acetonitrile over 30 minutes. Strands were checked for approximate mass on a Shimadzu Axima Performance MALDI-TOF instrument. Concentrations of purified products were determined by UV absorbance at $260 \mathrm{~nm}$ using a Thermo-Fischer NanoDrop. Cells were labeled by a 5-minute incubation in a 1 $\mu \mathrm{M}$ concentration of first the 'Adhesion' strand at room temperature followed by a 5minute incubation with $1 \mu \mathrm{M}$ of the 'Co-Anchor' strand at room temperature. Residual DNA strands were removed by washing the cells three times via pelleting and resuspension in calcium and magnesium free phosphate-buffered saline (PBS-CMF) (UCSF Cell Culture Facility).

\section{Cell Lines and Primary Cells}

Jurkat cells (Clone E6-1) ATCC TIB-152 were obtained from the ATCC and were grown in suspension using RPMI 1640 supplemented with $10 \%$ fetal bovine serum (v/v) (UCSF Cell Culture Facility). MCF10As were obtained from the lab of Professor Jayanta Debnath (UCSF) and were cultured as previously described ${ }^{2}$. HMEC cultures were provided by Dr. Martha Stampfer (LBNL) and cultured in M87 media supplemented with cholera toxin as previously described ${ }^{3}$. The specific specimen used in this study was $240 \mathrm{~L}$, which was obtained from the reduction mammoplasty of a 19 year old woman.

\section{Fabrication of PDMS Stamps}

PDMS stamps were produced from a photolithographically created master mold. SU-8 2075 (Microchem) was spun onto a silicon wafer to a thickness of approximately $100 \mu \mathrm{m}$. The wafer was briefly baked for 10 minutes on a hotplate set to $135^{\circ} \mathrm{C}$. The prebaked SU-8 was patterned with a negative mask (CAD/Art Services, Inc.) for 5 minutes. The wafer was subsequently baked for 1 minute on the hotplate set to $135{ }^{\circ} \mathrm{C}$ and then developed with agitation in 1-Methoxy-2-propyl acetate SU-8 developer (Microchem) for 15 minutes. Both sides of the wafer were washed with fresh 1-Methoxy2-propyl acetate to remove dissolved resist followed by washing with isopropyl alcohol. The wafer was subsequently baked on the hotplate for another 10 minutes, allowed to cool and placed with features face up into a $150 \mathrm{~mm}$ petri dish. 30 grams of 
polydimethylsiloxane (PDMS) elastomer (Sylgard® 184 Silicone Elastomer Kit, Dow Corning) was mixed with 3 grams of curing agent (10:1 ratio w/w) in a large weigh boat, mixed rapidly with a spatula until bubbles were apparent and poured into the petri dish, coating the wafer evenly. The petri dish with PDMS was then degassed until bubbles were no longer apparent, approximately 30 minutes. The PDMS was then baked for at least 3 hours at $60^{\circ} \mathrm{C}$. The PDMS was removed from the master by cutting with a disposable scalpel or razor blade. Stamps were created to fit a 24 well or 6 well dish by manually subdividing the PDMS elastomer with a razorblade.

\section{Fabrication of Microwell Arrays}

Microwell arrays were created by pipetting $400-500 \mu \mathrm{L}$ of molten agarose (Allstar Scientific, 490-050, Lot BB01013R0S) (3\% w/v) or gelatin (8\% w/v) (Knox Original Gelatin, unflavored) into each well of a 24 well plate and gently placing a PDMS stamp face down into the liquid in a manner so that the stamp features are in contact with the liquid, but not pressed down into the bottom of the well. For different sizes of stamps or vessels, the volume of agarose or gelatin scales with the surface area of the vessel. For example, $2 \mathrm{~mL}$ were used for a sample in a 6 well cell culture plate. The plate is then incubated at $4{ }^{\circ} \mathrm{C}$ until the agarose or gelatin solidifies which is apparent because it changes from translucent to opaque in appearance. The PDMS stamps are then gently removed by forceps and $500 \mu \mathrm{L}$ of PBS-CMF (UCSF cell culture facility) is added. In general, PDMS stamps were removed shortly before the microwells were used in order to avoid deformations that occur over time, particularly in the case of gelatin microwells.

\section{Procedure for Chemical Micromolding}

Cells are divided into two populations and stained with Cell Tracker Green CMFDA or Cell Tracker Red CMPTX (Thermofisher) as per manufacturers instructions. The two populations were labeled separately with complementary ssDNA strands as described above. Cells are mixed in a 1:1 ratio in a tube at a concentration of 1 million cells per $\mathrm{mL}$, added to the microwell array and placed promptly into a centrifuge. For microwells in a 24 well plate, we found 1 million Jurkats (500,000 of population 1 and 500,000 of population 2) filled the microwells with high occupancy, though this may need to be adjusted for cells of different sizes. Centrifugation speed was set to the slowest velocity at which a cell pellet was observed. For cells used in this study, we found $160 \mathrm{xg}$ for 4 minutes was sufficient. Typically this results in many full wells but also excess cells collecting around the edges of the microwell array. These excess cells are adhesive to each other as large aggregates visible by eye and easily removed by gently washing the edges of the array with PBS-CMF and $1 \mathrm{~mL}$ pipetteman. Cells were allowed to anneal in the microarray for 10 minutes and then incubated with $1 \mu \mathrm{M}$ capping strand for 10 minutes. Microtissues were recovered from agarose microwell arrays by gentle agitation with a $1 \mathrm{~mL}$ pipetteman with the pipet tip cut off. For gelatin microwell arrays, the plate is simply incubated in a tissue culture incubator set to $37^{\circ} \mathrm{C}$ for 20 minutes which returns the gelatin to liquid phase. The microtissues can be recovered from single cell debris by pulsing a centrifuge to $1000 \mathrm{~g}$ and immediately stopping in order to remove residual single cells and/or gelatin. For creating bilayered structures, microtissues were simply removed from microwell arrays in the presence of 
a high concentration of a third cell type. For a 24 well plate this was 5 million cells in a $500 \mu \mathrm{L}$ volume of PBS-CMF.

For the adhesion assay in the absence of ssDNA labeling (Figure S1), cells were allowed to adhere in agarose microwells for the noted amount of time before transfer.

\section{Cell Sorting and Staining}

HMEC cells were cultured in M87A media and used at passage 4. Single cell suspensions were prepared by trypsinization in $1 x$ TrypLE (Thermo), transferred to a 15 $\mathrm{mL}$ falcon tube and the trypsin quenched with fresh M87A. Cells were pelleted at $160 \mathrm{xg}$ for 4 minutes and resuspended in M87A at a concentration of $10^{6}$ cells $/ \mathrm{mL}$. Cells were stained with anti-human FITC-MUC1 1:50 (BD Clone HMPV) and anti-human PECALLA 1:200 (Biolegend Clone HI 10A) for 30 minutes on ice. Cells were washed three times in PBS to remove excess antibody and resuspended in $2 \%$ BSA in PBS. Pure populations of MEPs and LEPs were sorted from HMEC cultures using fluorescently activated cell sorting (FACS) on a FACSAriall (BD). LEPs were selected as $\mathrm{MUC}^{+} / \mathrm{CALLA}^{-}$and MEPs were selected as MUC1\% $1^{-} \mathrm{CALLA}^{+}$. Keratin staining was performed by fixing cultures in 4\% PFA for 20 minutes at room temperature. Samples were then treated with blocking buffer (10\% heat inactivated goat serum and $0.5 \%$ Triton X-100) for 1 hour at room temperature. Primary antibodies were diluted in blocking buffer and added overnight at $4^{\circ} \mathrm{C}$. After incubation in primaries, excess antibody was removed by $3 \times 15$ minute washes in blocking buffer. Fluorescently linked secondary antibodies were added at a dilution of 1:200 and incubated overnight. Following secondary incubation, samples were once again washed $3 x$ with blocking buffer before imaging. Primary antibodies used in this study were mouse anti-human Keratin-19 (Sigma Clone A53-B/A2; 1:50 dilution) and rabbit anti-human Keratin-14 (Thermo RB-9020-P (polyclonal); 1:50 dilution) to mark LEPs and MEPs, respectively. Secondary antibodies were Goat anti-mouse IgG Alexa 647 (Abcam ab150115, 1:200 dilution) and Goat anti-rabbit IgG Alexa 488 (Abcam ab150077, 1:200 dilution).

\section{Cell Imaging}

All images were collected using an inverted epifluorescence microscope (Zeiss Axiovert 200M, running Slidebook) with the exception of the confocal images in figure $2 \mathrm{D}$ and $2 \mathrm{E}$ which were acquired on a spinning disc confocal microscope (Zeiss Cell Observer Z1 with Yokagawa spinning disc, running Zen 2011). 


\section{Oligonucleotide Sequences:}

Anchor Strands:

1) A: 5'- 2 $_{24}$-GTA ACG ATC CAG CTG TCA CT(T) $)_{60}$ ACT GAC TGA CTG ACT GAC TG-3'

2) A': 5'-C 24 -GTA ACG ATC CAG CTG TCA CT(T) $)_{60}$ CAG TCA GTC AGT CAG TCA GT-3'

3) R: 5'-C $24^{-}$GTA ACG ATC CAG CTG TCA CT(T) 50 TATCCTATCCTGTGTGTGTGTATCCTATCC-3'

4) R': 5'- ${ }_{24}$ - GTA ACG ATC CAG CTG TCA CT(T) 50 GGATAGGATACACACACACAGGATAGG ATA-3'

Co-Anchor Strands:

1) Co-Anchor: 5' $T_{30}$ AGT GAC AGC TGG ATC GTT AC-C 24 3'

Fluorescent Strands:

Complementary to A: FAM-5'-(CAGT) $)_{5}-3^{\prime}$

Complementary to A': FAM-5'-(ACTG $)_{5}-3^{\prime}$

Complementary to R: FAM-5'-GGATAGGATACACACACACAGGATAGG ATA-3'

Complementary to R': FAM-5'-TATCCTATCCTGTGTGTGTGTATCCTATCC-3' 


\section{MALDI Mass Spectroscopy Data}

The purity and identity of fatty acid modified DNA oligonucleotides was confirmed by analytical HPLC and mass determination by MALDI-MS using a Shimadzu Biotech Axima Performance in positive linear mode with 3-hydroxy picolinic acid as the matrix. $2 \mu \mathrm{L}$ of matrix was spotted on the plate, followed by $2 \mu \mathrm{L}$ of a sample $(50 \mu \mathrm{M})$. The combined solutions were mixed and spots were allowed to dry under atmospheric pressure. Measurements were performed on at least 5 separate spots for each sample. Mass resolution decreased with the length of the Fatty Acid-conjugated oligonucleotides, yielding sharp peaks for oligonucleotides less than $10 \mathrm{kD}$, and broad peaks for oligos over $20 \mathrm{kD}$.

\begin{tabular}{lcccccccc} 
Molecule & $\begin{array}{c}\text { Actual } \\
\text { MW }\end{array}$ & \multicolumn{9}{c}{ Measured (M+H) } & & Average & StDev \\
& & 1 & 2 & 3 & 4 & 5 & & \\
A & 31025.46 & 31473.9 & 31471.79 & 31327.68 & $31,172.48$ & $31,267.0$ & 31342.6 & 131.2 \\
A' $^{\prime}$ & 31025.46 & 31391.8 & 31349.12 & 31511.32 & 31396.52 & 31311.54 & 31392.1 & 75.1 \\
R & 30971.44 & 31353.9 & 31425.93 & 31506.01 & 31339.06 & 31345.41 & 31394.1 & 71.9 \\
R' $^{\prime}$ & 31172.35 & 31506.0 & 31506.0 & 31470.5 & $31,396.95$ & $31,235.61$ & 31432.0 & 113.8 \\
Co-Anchor & 16112.39 & 16263.4 & 16258.1 & 16296.8 & 16254.8 & 16254.8 & 16265.6 & 17.8
\end{tabular}




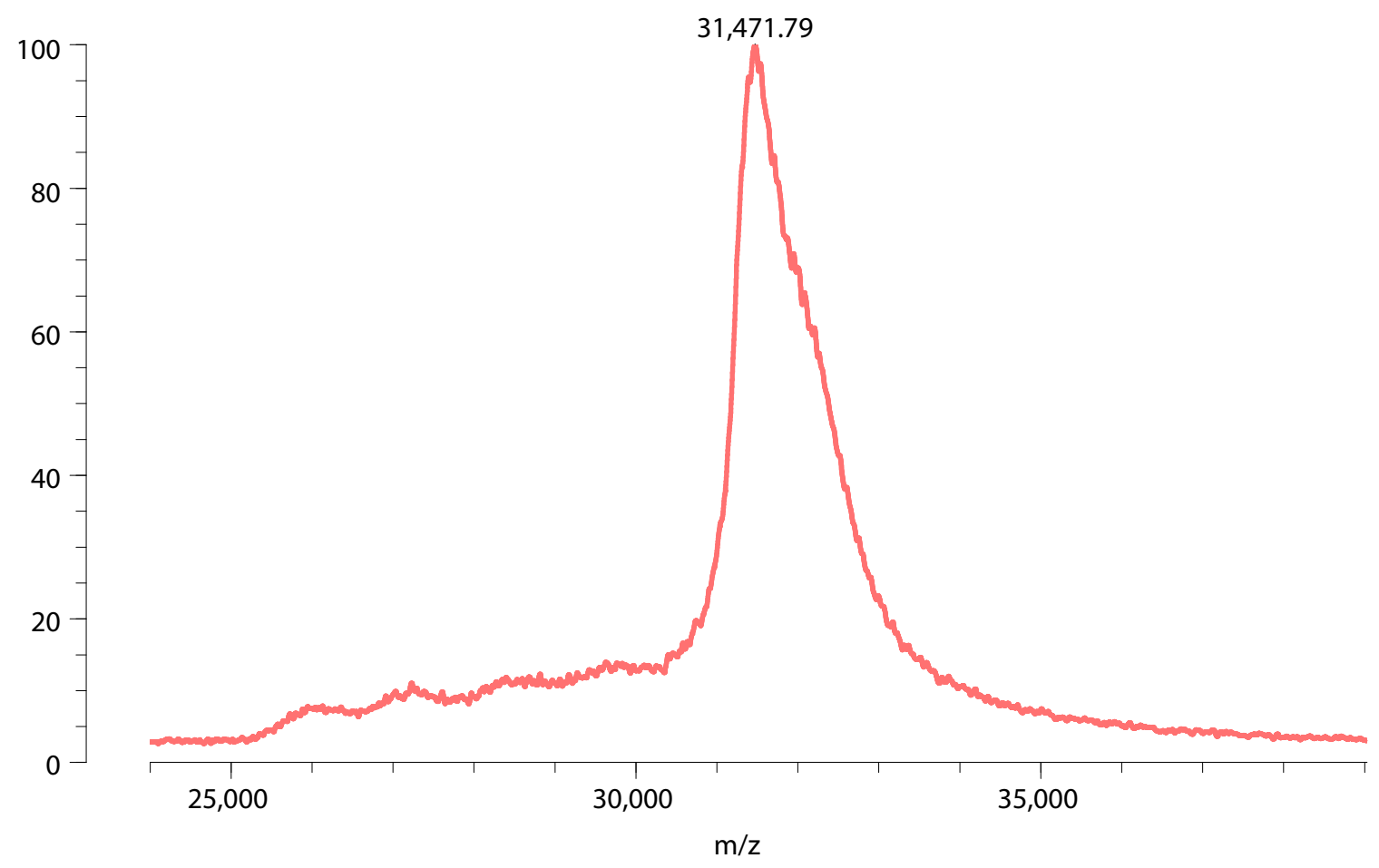

Representative MALDI trace of the A strand.

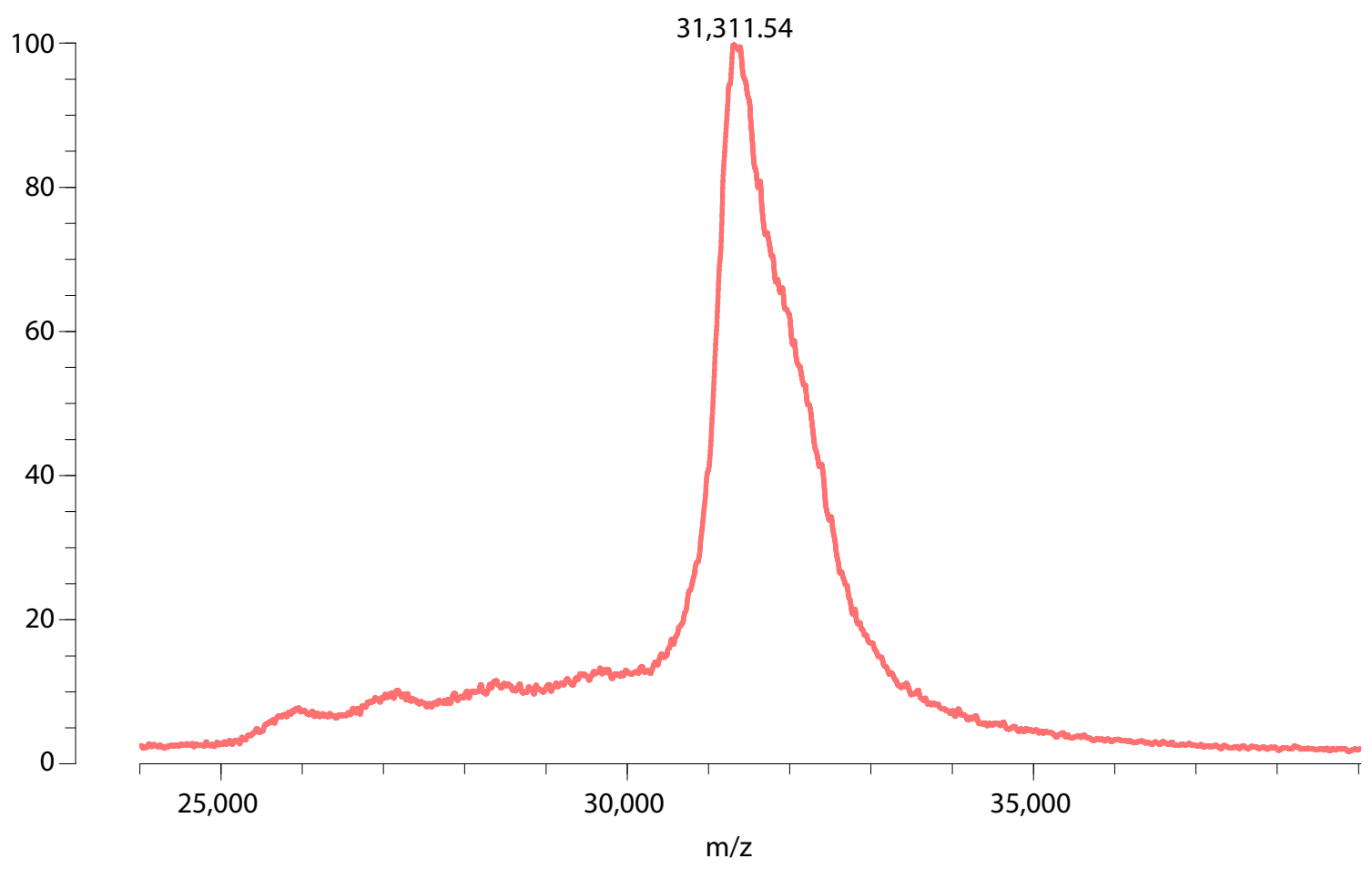

Representative MALDI trace of the A' strand. 


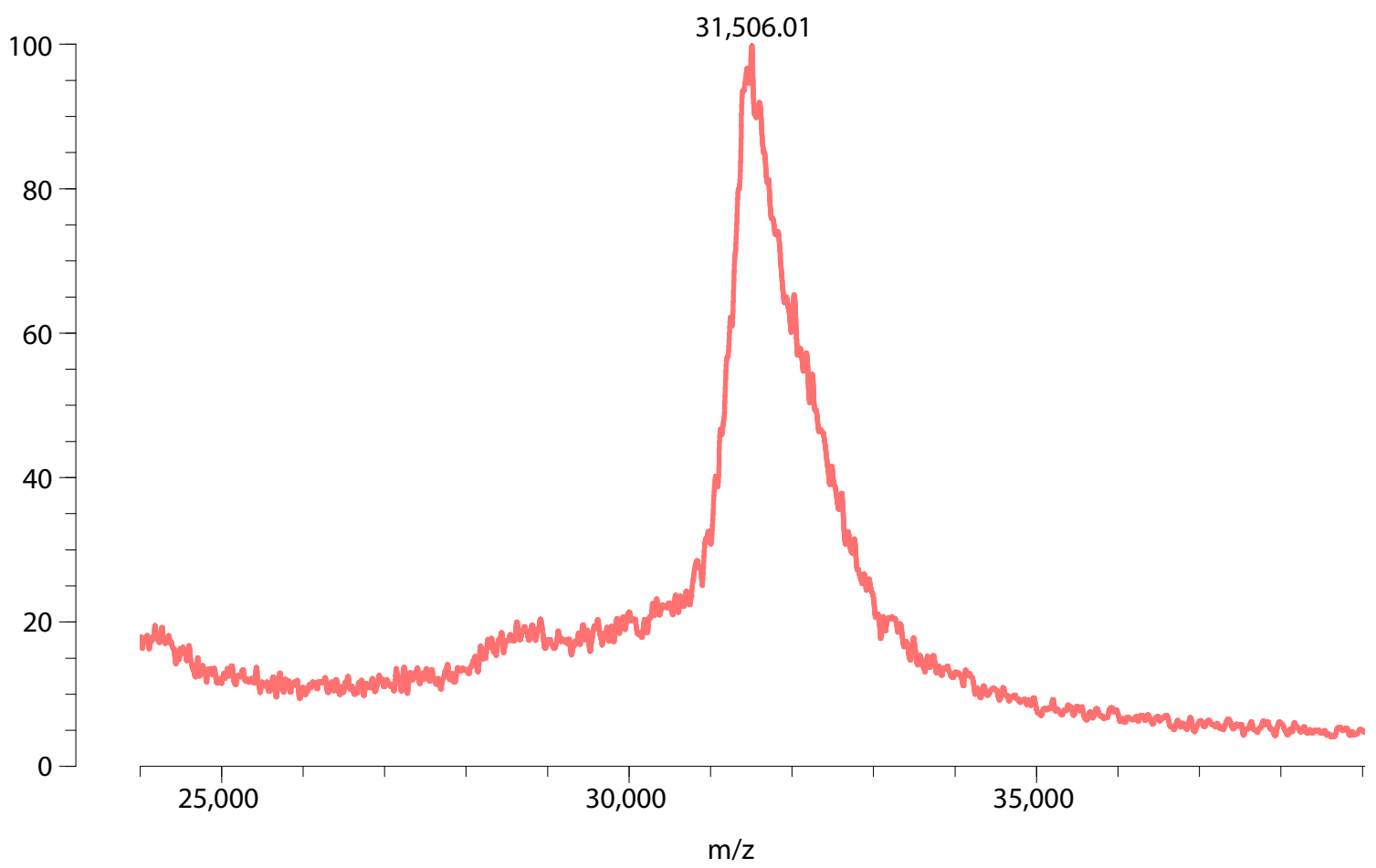

Representative MALDI trace of R strand.

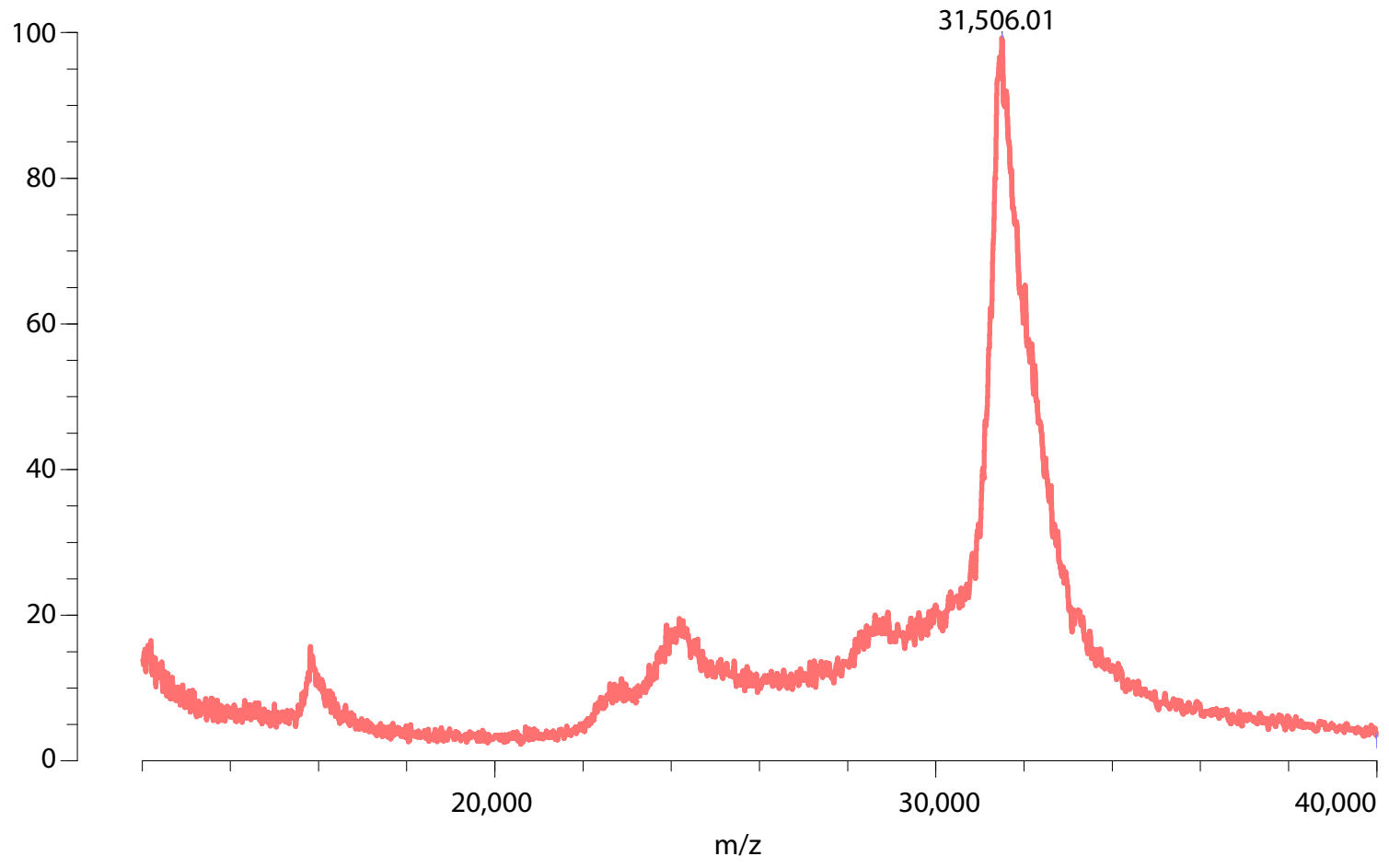

Representative MALDI trace of R' strand. 


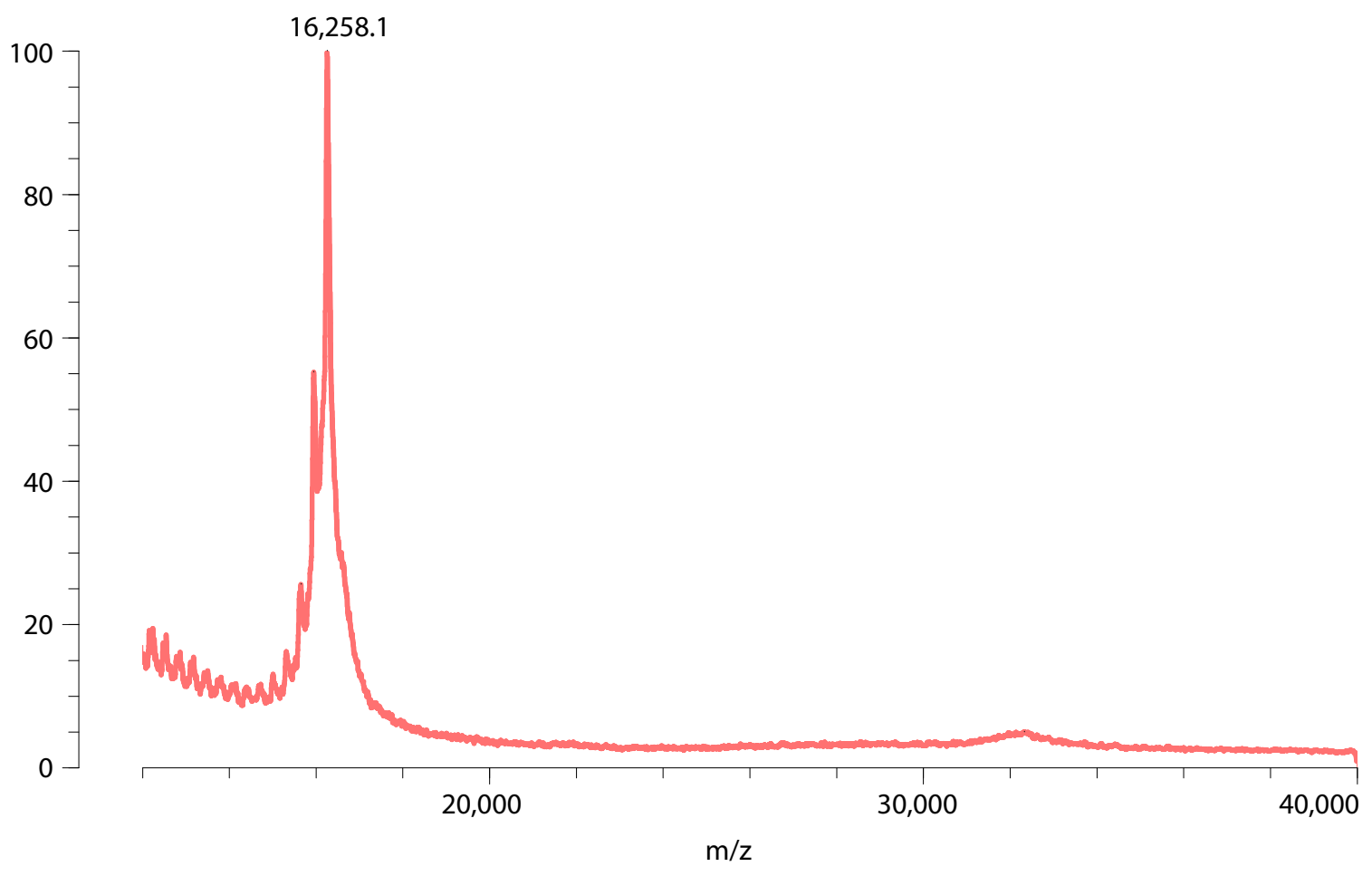

Representative MALDI trace of Co-Anchor. 


\section{A}
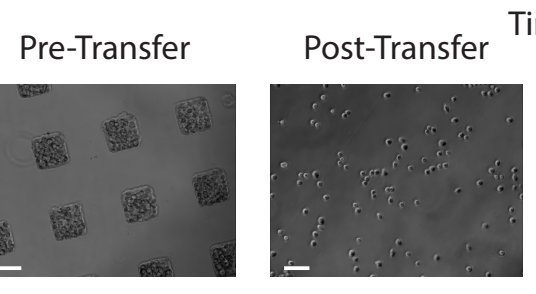

Time in Wells

(min)

0
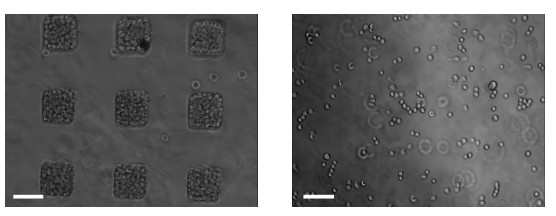

15
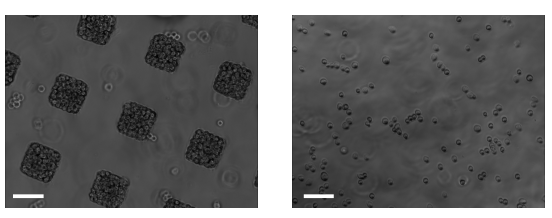

30
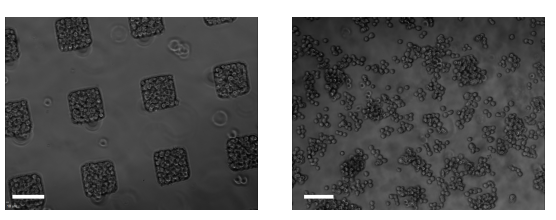

420
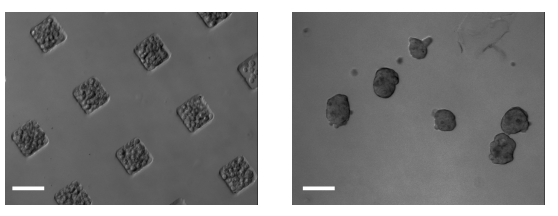

1020

B

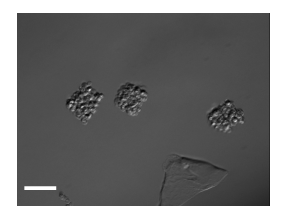

Figure S1. Adhesive Cells Coalescing In Microwells and Transferred Without DNA Do Not Retain Shape of Microwells. A. MCF10A cells, a cohesive epithelial cell line, were cast into square microwells, imaged and recovered from microwells at the indicated time. No adhesion occurred when transferred between 0 to 30 minutes, resulting in a spectrum of dissociated cells and small aggregates. By 420 minutes, some cell adhesion was observed, but the transferred cells were still composed of incomplete, rounded aggregates. By 1020 minutes, the cells are fully adhered, but also fully rounded with individual cells no longer readily apparent. This is in contrast to the complete MCF10A squares seen in B after only 10 minutes of DNAmediated adhesion in the wells followed by 10 minutes incubation with capping strand. Scale bars are $100 \mu \mathrm{m}$. Brightness of images was adjusted to be similar across samples. 
A

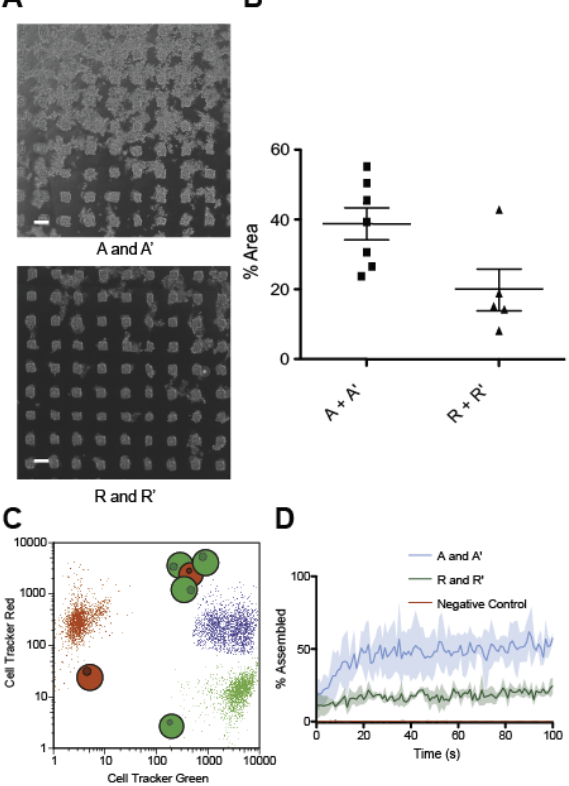

Figure S2. R/R' Show Slower Assembly Kinetics than A/A'.. A. Bright-field microscopy images depicting cell aggregates (grey) forming outside of wells labeled with the combination of A and A' oligonucleotides or the more heterogeneous R and R' oligonucleotides. Scale bars are $200 \mu \mathrm{m}$. B.

Quantification of the percentage of surface area obscured by aggregates outside of microwells. Each point is a separate microwell array and the line depicts the mean \pm 1 SEM. C. Flow cytometry scatter plot showing gating for quantifying aggregate formation. The FACS plot shows the flow positions of red cells alone, green cells alone and assembled cells. To measure the rate of assembly, red and green cells were complementarily labeled, mixed at a ratio of 1:50 respectively, and rapidly put onto the flow cytometer. D. Kinetics of Assembly. The plot shows the percentage of red cells moving from the red cloud (red cells only) to the blue cloud (red and green cells assembled) over 100 seconds. The plotted lines are the mean of $\mathrm{N}=3$ samples, the shaded area is \pm 1 SD. Negative cells were not labeled with any DNA. Brightness of images in A was adjusted to be similar between the two samples. 


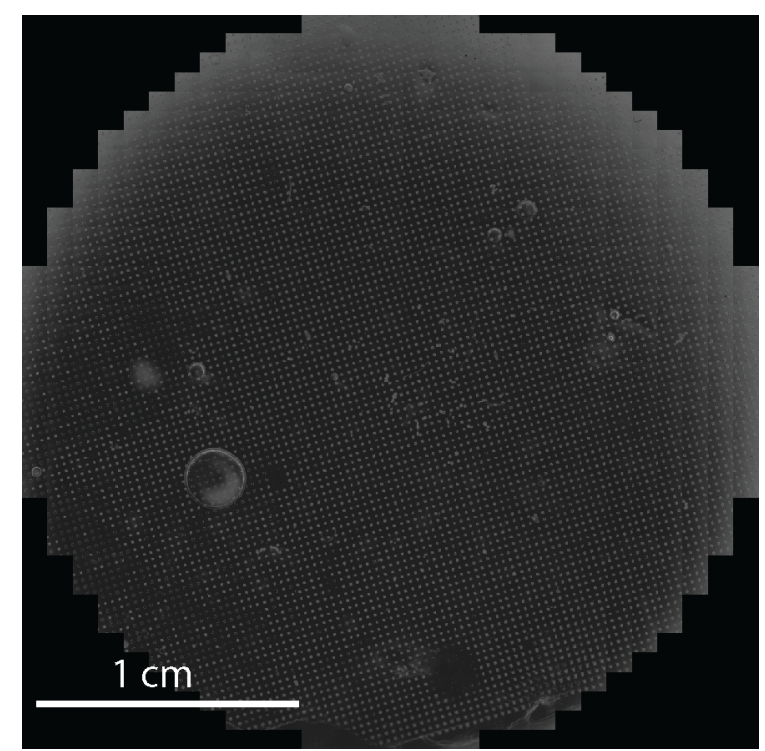

Figure S3. Scale-up of Chemical Micromolding With a Larger Stamp. A 10x tiled image across a large microwell array full of chemically

micromolded tissues. The array is approximately 107 microwells in diameter and comprises approximately 9,000 microwells in total. It was prepared in the same manner and using the same centrifugation time as smaller arrays with the number of cells increased proportionally to the increased surface area of the array. The array has approximately $80 \%$ occupancy with loss due to stamp imperfections as well as aggregates washed out of microwells during the removal of excess cells. This array was produced in 1 well of a 6 well plate. To scale up, one could simply use more wells of the plate or more plates. 


\section{R and A Labeling}

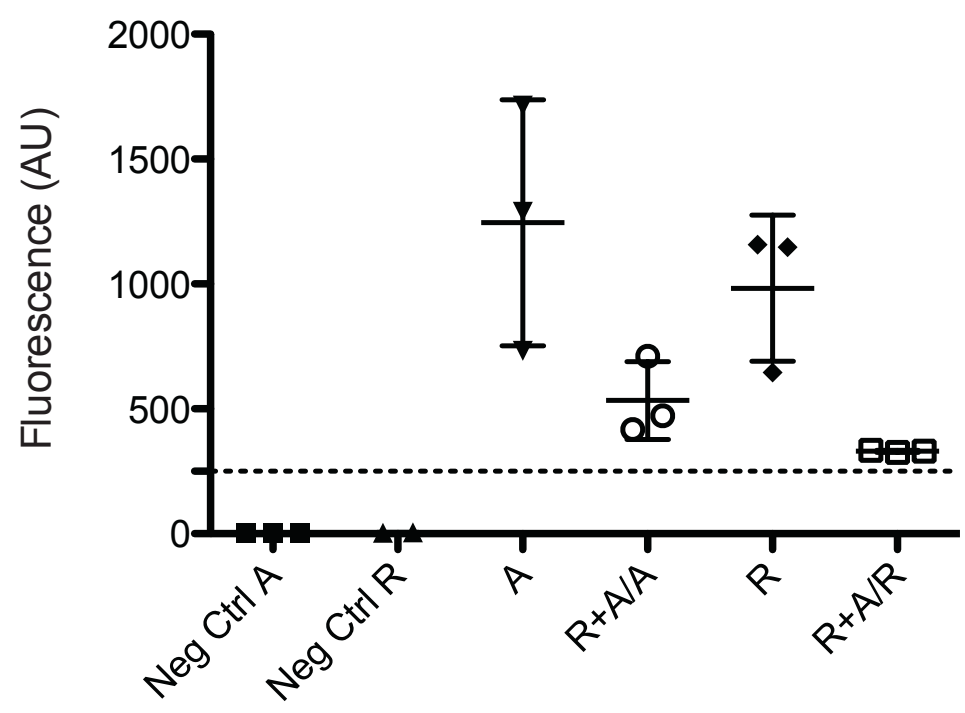

Figure S4. Efficiency of labeling cells with two unique strands of DNA

Cells were labeled with $1 \mu \mathrm{M}$ of both $\mathrm{A}$ and $\mathrm{R}$ strands simultaneously and $2 \mu \mathrm{M}$ of CoA-essentially a double dose of FA-DNA and then split in half and annealed to a FITC-oligo complementary to $A$ and $R$ respectively and fluorescence was measured by flow cytometry. There is a reduction in the

amount of fluorescence from each strand as compared to singly labeled controls indicating that less of each is incorporated. However, labeling was still above the minimum for cell-cell adhesion (dotted line). 
R Strand Adhesion Region

Heterogeneous Homogeneous Heterogeneous

\begin{tabular}{|c|c|c|c|}
\hline Capping Sequences 5'-3' & \# Bases & $\Delta \mathrm{G}\left(\mathrm{kcal} / \mathrm{mol}^{4}\right.$ & Graphical Depiction \\
\hline TACACACACACA & 12 & 11.9 & \\
\hline GGATACACACACACAGGA & 18 & 21.5 & GGAT \\
\hline TAGGATACACACACACAGGATA & 22 & 25.7 & TAGGAT \\
\hline GATAGGATACACACACACAGGATAGG & 26 & 31.7 & 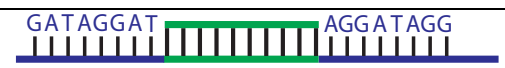 \\
\hline GGATAGGATACACACACACAGGATAGGAT & 30 & 37.1 & GGATAGGAT \\
\hline
\end{tabular}

Table S1. Map of R Strand Adhesion Region and Capping Strands as a Function of Length. The adhesion region of the R/R' strands has a central region of repeating 2 mers flanked by more heterogeneous regions. The repetitive region has been shown to have more rapid kinetics of annealing to complementary sequences in contrast to the more heterogeneous sequences. The capping strands are listed by sequence, number of bases, energy of binding, and a graphical depiction of where the capping strands bind. 


\section{References}

(1) Weber, R. J.; Liang, S. I.; Selden, N. S.; Desai, T. A.; Gartner, Z. J. Efficient Targeting of Fatty-Acid Modified Oligonucleotides to Live Cell Membranes through Stepwise Assembly. Biomacromolecules 2014, 15 (12), 4621-4626 DOI: 10.1021/bm501467h.

(2) Debnath, J.; Muthuswamy, S. K.; Brugge, J. S. Morphogenesis and oncogenesis of MCF-10A mammary epithelial acini grown in threedimensional basement membrane cultures. Methods 2003, 30 (3), 256-268 DOI: 10.1016/S1046-2023(03)00032-X.

(3) Garbe, J. C.; Bhattacharya, S.; Merchant, B.; Bassett, E.; Swisshelm, K.; Feiler, H. S.; Wyrobek, A. J.; Stampfer, M. R. Molecular Distinctions between Stasis and Telomere Attrition Senescence Barriers Shown by Long-term Culture of Normal Human Mammary Epithelial Cells Cancer Research 2009, 69 (19), 7557-7568 DOI: 10.1158/0008-5472.CAN-09-0270.

(4) Kibbe, W. A. OligoCalc: an online oligonucleotide properties calculator. Nucleic Acids Research 2007, 35 (Web Server), W43-W46 DOI: $10.1093 /$ nar/gkm234 